\title{
Application of Characteristic Model-Based Principal Component Analysis in Optimization of Flowmeter Parameters
}

\author{
Wenping Jiang $\mathbb{D}^{1},{ }^{1}$ Zhencun Jiang $\mathbb{D}^{1},{ }^{1}$ Lingyang Wang, ${ }^{1}$ Jun $M i n,{ }^{2}$ Yi Zhu, ${ }^{3}$ \\ Zhonghui Wang, ${ }^{3}$ Lizhuang Tang, ${ }^{3}$ and Limin Sun $^{3}$ \\ ${ }^{1}$ Shanghai Institute of Technology, School of Electrical and Electronic Engineering, No. 100 Haiquan Road, Fengxian District, \\ Shanghai 201418, China \\ ${ }^{2}$ Tongji University, No. 1239 Siping Road, Yangpu District, Shanghai 200082, China \\ ${ }^{3}$ Bitobar Company, No. 265 Lingdong Street, Tieling, Liaoning 112000, China
}

Correspondence should be addressed to Wenping Jiang; jiangwenping@sit.edu.cn

Received 31 March 2021; Revised 8 June 2021; Accepted 4 August 2021; Published 20 August 2021

Academic Editor: Zhiwei Gao

Copyright $(92021$ Wenping Jiang et al. This is an open access article distributed under the Creative Commons Attribution License, which permits unrestricted use, distribution, and reproduction in any medium, provided the original work is properly cited.

\begin{abstract}
In complex industrial processes, it is necessary to perform modeling analysis on some industrial systems and find and optimize the factors that have the greatest impact on the results, in order to achieve the optimization of the industrial systems. However, due to the high-level nature or complex working mechanism of complex industrial systems, traditional principal component analysis methods are difficult to apply. Therefore, this paper proposes a characteristic model-based principal component analysis (CMPCA) to perform principal component analysis on complex industrial systems. The differential pressure flowmeter is taken as an example to verify the effectiveness of the method. Flowmeter is an indispensable instrument in measurement, and its accuracy depends on its own structural parameters. However, the measurement accuracy of some flow meters is not high, and the measurement error is large, which affects the normal industrial production process. This method is used to analyze the influence of the structural parameters of the flowmeter on its measurement accuracy, and the four most important structural parameters are found and optimized. The measurement error of the Bitoba flowmeter is reduced from $1 \%$ to $0.2 \%$, and the measurement repeatability is reduced from 0.3 to 0.06 , which proves the effectiveness of the method.
\end{abstract}

\section{Introduction}

In the industrial production process, with the increase of testing means, the production line can provide a large number of production data for the application of engineers. Meanwhile, with the upgrade of the CPU, data-driven modeling and control become possible.

For complex industrial systems [1], traditional dynamics modeling is difficult to apply due to its high order or complex working mechanism, and even if it is applied, mathematical methods are needed for downscaling modeling: For the simplification of single-input single-output models of large power systems, a lot of people study model simplification method based on SVD-Krylov subspace projection methods, which aims to rely on singular value decomposition and Krylov subspace methods for order reduction system. The intelligent method is mainly applied in neural networks, fuzzy logic, genetic algorithm, etc. This method can solve the partial open-loop control problem without the need for an accurate dynamic model. It mainly constructs the model form by experience and then obtains the model through parameter identification. This method is limited in industrial production because it cannot guarantee the effect of online control. Yichuan Fu and Zhiwei Gao et al. proposed classifying the actuator and sensor faults of wind power equipment based on fast Fourier transform (FFT) and uncorrelated multilinear principal component analysis (UMPCA), which were tested and verified on wind turbines [2]. Due to the complex working mechanism of intermittence and continuity in the industrial system, the variables are often nonlinearly correlated. The traditional data-based PCA has a large calculation error in this case, because of the 
linear relationship between the assumed variables, so it is not suitable for dynamic nonlinear working mechanisms.

The characteristic modeling method was proposed by Wu Hongxin Academician of the Chinese Academy of Sciences in the 1980s [3], which broke through the original framework of modeling controlled objects and provided a new idea for the modeling of high-order complex systems with unknown parameters and orders. It has laid a theoretical foundation for the realization of low-order controller design for some high-order systems. After more than 30 years of research and accumulation, important progress has been made in both theory and application. At present, the problem of characteristic modeling for linear time-invariant systems and affine nonlinear systems has been basically solved [4]. This method has been successfully applied to hundreds of systems in aerospace and industrial control fields and has important practical application prospects.

Flow measurement is an important guarantee for industrial automation. As the requirements for measurement accuracy in the industrial production process gradually increase, people's demand for flow measurement is also increasing. A lot of investment has been used to improve the automation level of the plant, which is more reflected in the field data collection and real-time monitoring. In the energy industry or in the chemical-pharmaceutical industry, high-precision flow meters are required [5-7]. Taking Bitoba Flowmeter as an example, it is a differential pressure flowmeter; this kind of flowmeter has a good application prospect in petroleum, metallurgy, water supply, and other fields, especially in the large-diameter industry. However, there are still two problems. (1) The flowmeter cannot fully adapt to the real situation of the factory due to the calibration of the flow coefficient is completely determined by the factory test. (2) The measurement accuracy needs to be further improved and the measurement reproducibility is expected to be reduced to an ideal level [8-12].

To address these two problems, a characteristic modelbased principal component analysis method is proposed in this paper, and the structural parameters of the flowmeter are optimized using this method. A three-dimensional simulation model was performed on the flowmeter, and the optimized structural parameters were tested in the simulation environment and verified in actual industrial production. The second part introduces the principle of the characteristic model, the principal component analysis method based on the characteristic model, and the flowmeter simulation design method. The third part is the result of the flowmeter simulation modeling and the results of the principal component analysis method based on the characteristic model on flowmeter parameter analysis. The fourth part is the conclusion.

\section{Materials and Method}

2.1. Characteristic Model-Based Principal Component Analysis. For the process control systems that widely existed in industrial processes and cannot be accurately modeled, the characteristic model method can identify the nonlinear relations existing in the system through data identification and decouple the coupling relations between various variables. For the same structure, no matter how the initial value is selected, the system parameters will converge to a relatively fixed value. This is the difference between the characteristic model method and intelligent algorithms such as neural networks. Therefore, the characteristic model method can accurately determine the system's principal components, thus providing a practical and effective method for the modeling and control of the system.

The so-called characteristic model-based principal component analysis is the model built according to the dynamic characteristics, environmental characteristics, and control performance requirements of the object, and it finds the principal components of the object, not only using the precise dynamic equations of the object to model. The characteristic model is different from the higher-order model in order reduction, but the relevant information of the higher-order model is compressed into several characteristic parameters without losing. In the dynamic process, the output of the characteristic model is equal to that of the real object. In the stable state, the outputs are more equal, and the range of coefficients of the characteristic model can be determined in advance.

The main features of the characteristic model-based principal component analysis include the following aspects:

(1) The characteristic model compresses the main information of the higher-order model into several characteristic parameters. In general, the characteristic model can be described by the slow timevarying difference equation

(2) The obtained characteristic model expression must have lower order than the original dynamic model, which is easy to realize in the industrial field

(3) The order and form of the characteristic model mainly depend on the control performance requirements, in addition to the need to consider the object characteristics

(4) In the stable state, the output of the object characteristic model and the actual object are equal. If the system is given the same input signals, they are also equivalent in terms of output; that is, they can be kept within the allowable output error in an unstable state

For systems whose physical characteristics are clear but difficult to describe with dynamic equations, we can directly establish the characteristic model expression between the control variables represented by physical characteristics and their physical characteristics output and extract the principal components.

The transfer function of the high-order linear time-invariant system commonly seen in engineering is as follows:

$$
G(s)=\frac{b_{m} s^{m}+b_{m-1} s^{m-1}+\cdots+b_{1} s+b_{0}}{s^{n}+a_{n-1} s^{n-1}+\cdots+a_{1} s+a_{0}} .
$$

It can be simplified into the following form: 


$$
\begin{aligned}
G(s)= & \frac{k_{v}}{s^{2}}+\sum_{i=1}^{m} \frac{k_{i}}{s+\lambda_{i}}+\sum_{i=1}^{m}\left(\frac{k_{p+i}}{s+\lambda_{p+i}}+\frac{\bar{k}_{p+i}}{s+\bar{\lambda}_{p+i}}\right) \\
& +\sum_{i=1}^{p} \frac{k_{\omega_{i}}}{\left(s+\omega_{i}\right)^{2}} .
\end{aligned}
$$

Equation (2) can also be described by the higher-order timevarying difference equation, as shown in equation (3). However, if the coefficients of the difference equation change in real time, then the higher-order time-varying difference equation can be rewritten into a time-varying difference equation of order 2 . The order of the difference equation is determined according to the actual situation.

For any $n$-order linear time-invariant system $G(s)$ that can be decomposed according to equation (2) to achieve position retention or position tracking control, under the condition that a certain sampling period is satisfied, it can be expressed in the form of the second-order time-varying difference equation as follows:

$$
\begin{aligned}
y(k+1)= & \alpha 1(k) y(k)+\alpha 2(k) y(k-1)+\beta 0(k) u(k) \\
& +\beta 1(k) u(k-1) .
\end{aligned}
$$

When the object $G(s)$ is stable or contains integral links, then one has the following:

(1) The coefficients of equation (3) are slow-varying

(2) The range of coefficients can be determined in advance

(3) Assuming that the sampling period can ensure that the error of the output is within the allowed range, the output of the characteristic model can be used to replace the output of the actual system under the condition that the input is the same, and the two are equal; in the stable state, the static gain of equation (3) is equal to the static gain of equation (2)

(4) When the static gain $D=1$, the system tends to be stable and the sum of the coefficients of the difference equation is equal to 1 ; then

$$
\alpha 1(k)+\alpha 2(k)+\beta 0(k)+\beta 1(k)=1 .
$$

(5) The object has integral link time:

$$
\alpha 1(k)+\alpha 2(k)=1
$$

For the establishment of first-order and second-order characteristic models for a continuous system, its mathematical description can be expressed by the following equation:

$$
\dot{y}=f(y, u) \text {. }
$$

In sample equation (6), the sampling period is $\Delta t$. The general second-order characteristic model is as follows:

$$
y(k+1)=f_{1}(k) y(k)+f_{2}(k) y(k-1)+g_{0}(k) u(k) .
$$

The first-order Taylor expansion can also be used to obtain

$$
y(k+1)=f_{1}(k) y(k)+g_{0}(k) u(k) .
$$

For the general dynamic equations, the unknown system can also be directly constructed according to the characteristic mechanism, as shown in equations (7) and (8).

2.2. Flowmeter Structure. The Bitobar flowmeter's physical diagram is shown in Figure 1.

Its composition principle is shown in Figure 2.

The most important factor which affects the measurement accuracy is the part of the pressure head. The threedimensional diagram of the pressure head is shown in Figures 3(a)-3(c).

The structural parameters of the indenter are mainly as follows:

(1) The angle of full pressure surface

(2) Static pressure plane angle

(3) The distance from the centerline to the full pressure hole

(4) The distance from centerline to static pressure hole

(5) The height of the static hole

We build a mathematical model between the data and the test model based on the data provided by the manufacturer. The mathematical model includes the following parameters: standard volume, standard mass, flow rate, pressure, Reynolds number, test current, flow coefficient, error, and repeatability.

2.3. Working Principle of Flowmeter. The calculation formula of the volume flow of fluid is shown in

$$
q v=\sqrt{\frac{2 \Delta P}{\rho}},
$$

where $q v$ is the volume flow rate and its unit is $\mathrm{m}^{3} / \mathrm{s} . \Delta P$ is the pressure difference between total pressure and static pressure. The density of a medium fluid is $\rho$ and $\mathrm{kg} / \mathrm{m}^{3}$ is its unit. Let $A=\pi D^{2} / 4$; equation (9) can be derived as equation (10). The cross-sectional area of the pipe's unit is $\mathrm{m}^{2}$ and is denoted as A. The unit of the inner diameter of the pipe is $\mathrm{mm}$, and it is expressed as $D$. $a$ is a dimensionless flow coefficient.

$$
\alpha=7.90484 \frac{q^{v}}{D^{2}} \sqrt{\frac{\rho}{\Delta P}} .
$$

In industry, the flow coefficient $a$ must be measured by the flowmeter using the flow standard device, so when the flowmeter becomes the main body of this study, the flow coefficient $\alpha$ is a known quantity. The volume flow in the pipeline can be calculated by calculating the measured pressure difference, density, and cross-sectional area as equation (9). The equation for calculating the repeatability of verification point $I$ is shown in 


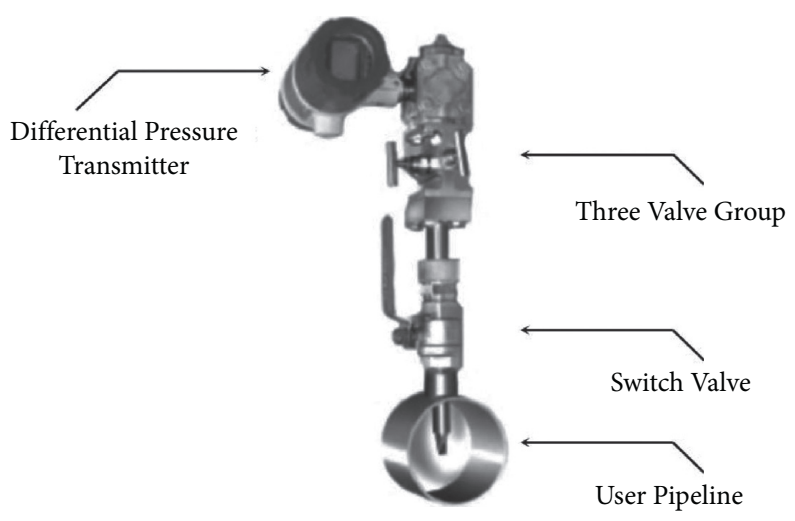

FIgURE 1: Flowmeter physical map.

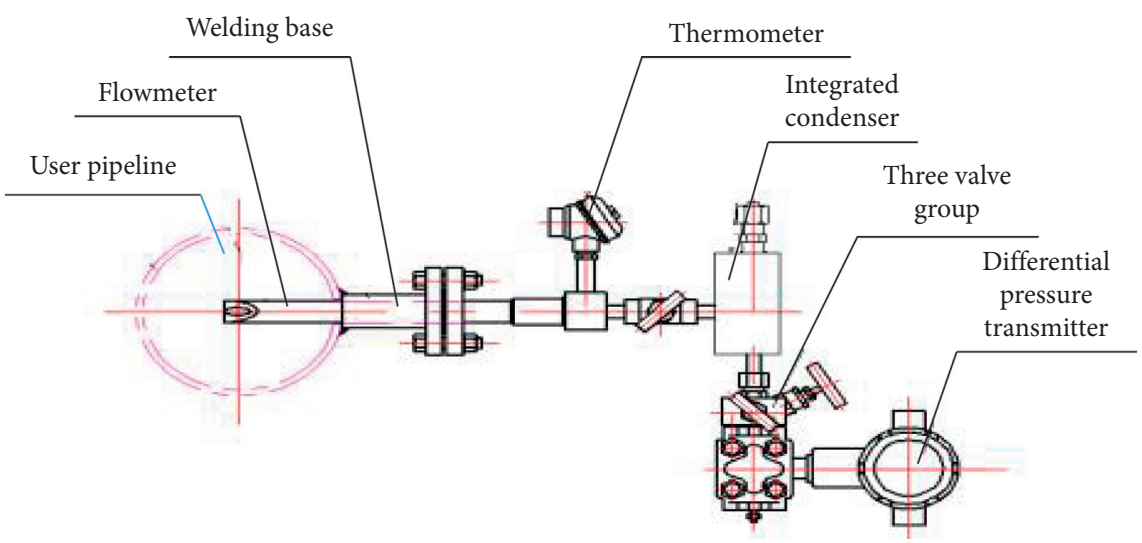

FIgURE 2: Flowmeter composition schematic.

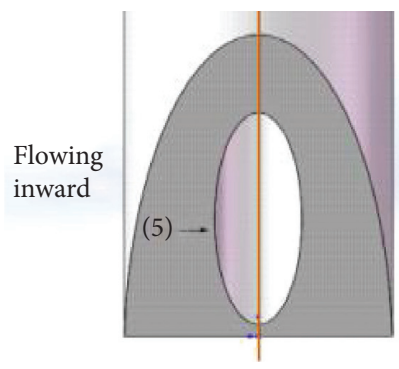

(a)

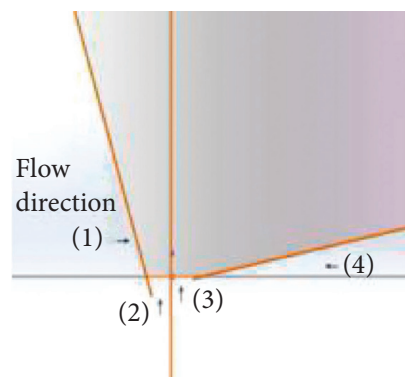

(b)

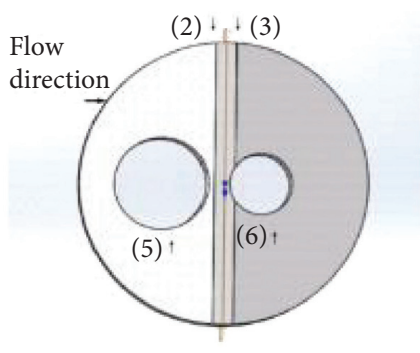

(c)

Figure 3: Three views of the flowmeter head section. (a) The flowmeter pressure head's front view. (b) The flowmeter pressure head's end view. (c) The flowmeter pressure head's vertical view.

$$
\left(E_{r}\right)_{i}=\frac{1}{\alpha i}\left[\frac{1}{(n-1)} \sum_{i=n}^{n}\left(\alpha_{i j}-\alpha_{i}\right)^{2}\right]^{1 / 2} \times 100 \% .
$$

The equation used to calculate the flow coefficient of the flow meter is shown in

$$
E=\frac{(\alpha i) \max -(\alpha i) \min }{(\alpha i) \max +(\alpha i) \min } \times 100 \% .
$$

The calculation equation of the linear error of the flow meter is shown in

$$
\alpha=\frac{(\alpha i) \max +(\alpha i) \min }{2} .
$$

Flow coefficient $\alpha_{i}$ reflects the characteristics of the device. Its size and linear characteristics are related to the shape, size, industrial conditions, and pipe diameter of the equipment. For the Bitobar differential pressure flowmeter, the flow coefficient must be determined first. After the determination of the flow coefficient, the real flow value can be obtained and the indication error can be determined. 
2.4. Flowmeter Simulation. Working conditions have a great influence on the accuracy of the flowmeter, so are the structural parameters. Now, the flowmeter's accuracy will change with different pipe diameters, liquids, or pipe filling degrees. It is necessary to design the structural parameters previously and simulate a test for the flowmeter [13-18].

(1) SolidWorks: SolidWorks, the most wildly used drawing software in engineering, can build a flowmeter's threedimensional model. What's more, the software has powerful functions, simple operations, and a rich conversion interface. We need to use it for modeling

(2) HyperMesh: HyperMesh can be used for grid dividing. Nowadays, there are many kinds of software for grid diving, such as ICEM and ANSA. Compared with HyperMesh, ICEM and ANSA both have disadvantages. Firstly, ICEM cannot generate high-quality prism meshes in the boundary layer. Secondly, the degree of freedom of ANSA in generating meshes is not as high as HyperMesh. HyperMesh can manipulate the grid precisely. And it can delete and merge nodes, so HyperMesh is selected to divide the grid

(3) Gambit: Gambit is used to process the model's boundaries. After being processed by HyperMesh, the model was cut into many small squares. Using gambit software, the boundary of these grids can be assigned, and the parameters inputted should be consistent with the actual data. Then, we define the flow rate, pressure, and other parameters to prepare for the following fluid calculations

(4) Fluent: At present, Fluent is the most widely used software in fluid calculation. It supports discontinuous mesh, hybrid mesh, dynamic deformable mesh, and sliding mesh. It has powerful performance and can meet the needs of different conditions and can perform complex fluid calculations

In this paper, the $\kappa-\varepsilon$ RNG model is used instead of the standard $\kappa-\varepsilon$ model [19-21]. RNG theory provides an analytical formula to consider viscous flow at a low Reynolds number. In a wide flow range, the $\kappa-\varepsilon$ model is more reliable than the standard $\kappa-\varepsilon$ model and more accurate than the $\kappa-\varepsilon$ model. The $\kappa-\varepsilon$ RNG model is suitable for various flow types, especially in the simulation of circular jet and plane jets, which can make the fluid flow simulation more reasonable. The $\kappa-\varepsilon$ RNG model is used to simulate the outflow coefficient. The average error of numerical simulation is about $5 \%$, and the maximum value is less than $8 \%$.

At the same time, the SIMPLE algorithm [22-25] is used and it takes the pressure as the basic variable. There are five steps in the process of the SIMPLE algorithm, shown in Figure 4.

\section{Experimental Results and Analysis}

3.1. Flowmeter Model Simulation Results. Firstly, the simulation model is validated by the factory test data. Secondly, more than 70 groups of data can be obtained when simulating each feature number from 10 to 15 times. Thirdly, the influence of each characteristic parameter of the flowmeter is analyzed when the preliminary database is established. Thus, the optimal model is determined. Finally, the simulation block diagram is shown in Figure 5.

Let the pressure be equal to $185000 \mathrm{~Pa}$ and the mass velocity is $84.0277 \mathrm{~kg} / \mathrm{s}$. The $\kappa-\varepsilon$ RNG model is selected, iterated 50 times until convergence. The final simulation result is shown in Figure 6. The value of the simulated pressure difference $\Delta P$ is 2.568104 , while the real data given by the manufacturer is 2.5423104 . Therefore, the error between the algorithm adopted in this experiment and the actual results is shown in equation (14) with the first set of parameters.

$$
E=\frac{2.568-2.5423}{2.5423}=1.1089 \% .
$$

The result shows that the simulation error is $1.1 \%$. Similarly, multiple sets of real data on-site are used to verify the accuracy of the simulation method, and the maximum error is $8 \%$. The smaller the diameter of the static pressure hole is, the smaller the measurement error will be. However, the static pressure hole will be blocked by impurities which need to be cleaned up frequently when the static pressure hole is too small. Therefore, different diameters are formulated to ensure the accuracy and continuity of production according to different media. For different velocities, the position of the average velocity is 0.24 times the radius of the pipe wall, diameter, and roughness in the process of measurement.

\subsection{Characteristic Model-Based Principal Component Anal-} ysis Application Results. Through the analysis of the flowmeter structure, five structural parameters that affect the measurement accuracy of the flowmeter are selected, and characteristic model-based principal component analysis is used to further principal component analysis of these five parameters.

Structural fitting data is based on the following characteristic model.

$$
\begin{aligned}
y(k+1)= & f_{1}(k) u_{1}(k)+f_{2}(k) u_{2}(k) \\
& +f_{3}(k) u_{3}(k)+f_{4}(k) u_{4}(k) \\
& +f_{5}(k) u_{5}(k),
\end{aligned}
$$

where $f_{i}(k)$ is the characteristic model coefficient, $u_{i}(k)$ is the structural parameters, and $y(k+1)$ is the system output $\Delta P$. The identification method we selected is the gradient method. The main ideas of the gradient method are as follows. Firstly, start at one point and the direction where the function drops fastest is taken as the search direction. Then, the direction where the function value at any point drops fastest is its negative gradient direction. Finally, the $N$-dimensional problem is transformed into a one-dimensional problem, and the one-dimensional search method is used to find the optimization along the negative gradient direction. The gradient method can be used to solve the nonlinear equations with real coefficients, such as a group of roots belonging to $f_{i}\left(x_{1}, x_{2}, \ldots x_{n}\right)=0, i=1,2, \ldots, n$. The gradient method needs to define an objective function firstly: 
Correct the pressure field

\begin{tabular}{|c|c|c|c|c|}
\hline & & & & \\
\hline fiel & Pressure field & Momentum & Velocity field & Continuity \\
\hline
\end{tabular}

(a) The distribution of the initial pressure field is assumed. (b) Momentum equation is solved by the pressure field Then the velocity field is obtained. (c) Solving continuity equation by the velocity field and correct the pressure field.

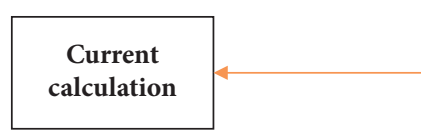

(e) The current calculation

is judged by convergence. (d) Solving the turbulence equation and the other equations.

FIgUre 4: The process of the SIMPLE algorithm.

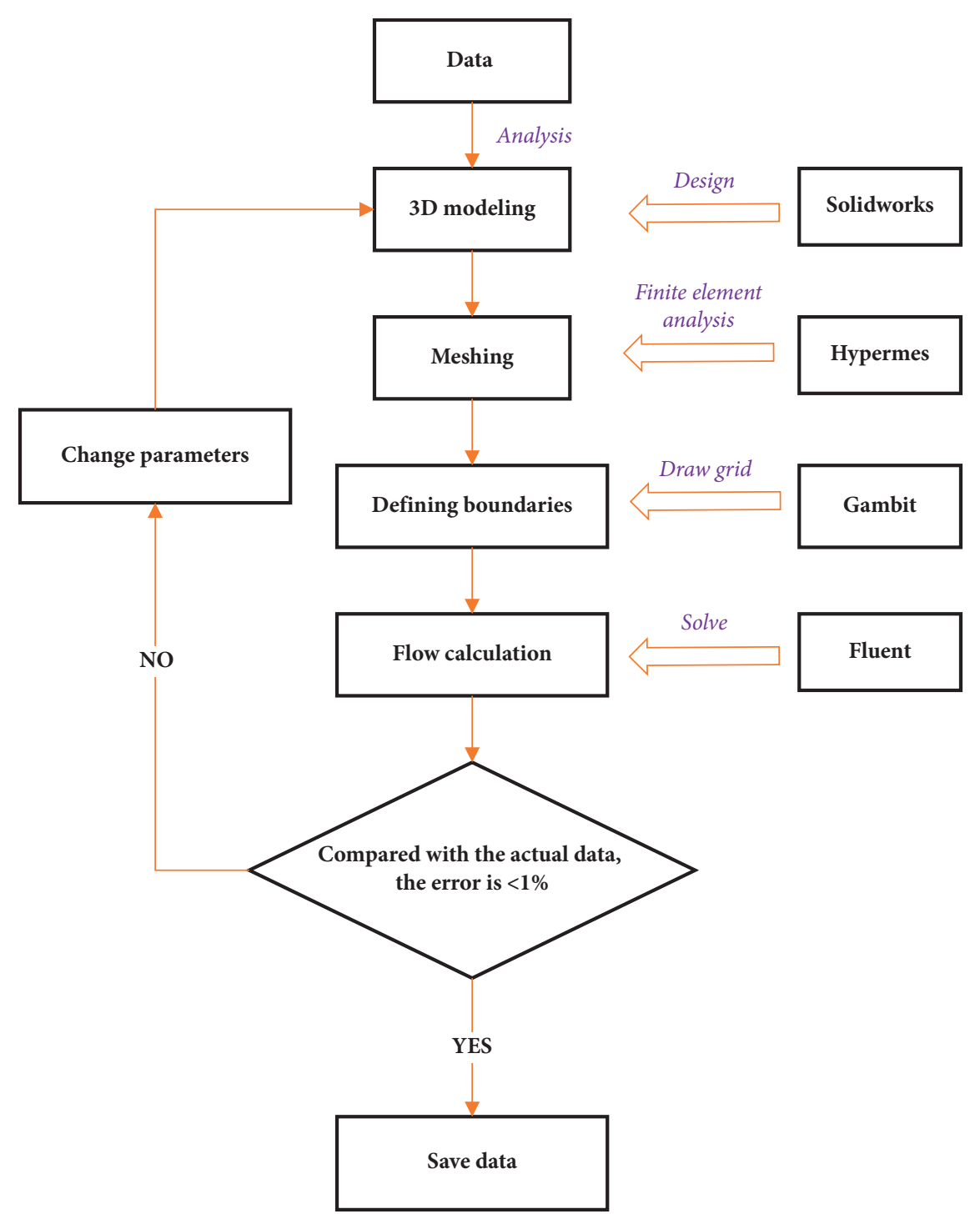

FIGURE 5: Simulation block diagram. 

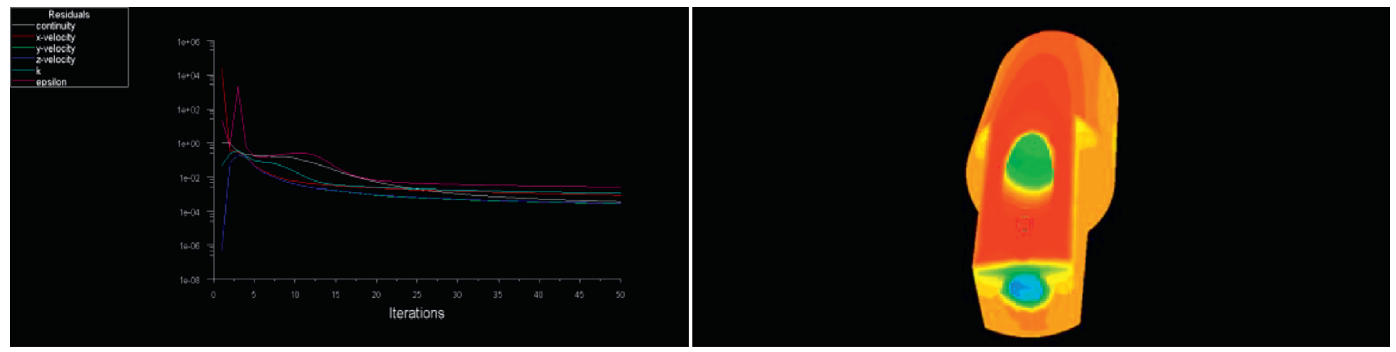

FIGURE 6: Simulation results.

$$
\Phi\left(x_{1}, x_{2}, \Lambda, x_{n}\right)=\sum_{i=1}^{n} f_{1}^{2}\left(x_{1}, x_{2}, \Lambda, x_{n}\right) .
$$

Secondly, we look for the minimum value of the objective function $\phi=\sum_{i=1}^{n} f_{1}^{2}$ among $x_{1}, x_{2}, \ldots x_{n}$. If we get a set of solutions: $x_{1}{ }^{k}, x_{2}{ }^{k}, \ldots x_{n}{ }^{k}$ at the $k$ step $(k=0,1,2, \ldots)$, then making $\Phi=\left(x_{1}{ }^{k}, x_{2}{ }^{k}, \ldots x_{n}{ }^{k}\right)<\varepsilon$, where $x_{1}{ }^{k}, x_{2}{ }^{k}, \ldots x_{n}{ }^{k}$ is a set of efficient solutions of the original nonlinear equations under certain precision conditions. The iterative steps of the gradient method are as follows.

Step 1. Give an initial value $x_{1}^{0}, x_{2}^{0}, \ldots x_{n}^{0}$ (the initial value cannot be 0 completely) and set up the roots obtained in step $k$ which are $x_{1}{ }^{k}, x_{2}{ }^{k}, \ldots x_{n}{ }^{k}$.

Step 2. Calculate the value of the objective function $\Phi=\left(x_{1}^{k}\right.$, $\left.x_{2}^{k}, \ldots x_{n}^{k}\right)$.

Step 3. If $\Phi=\left(x_{1}^{k}, x_{2}^{k}, \ldots x_{n}^{k}\right)$, we can think that $x_{1}^{k}, x_{2}^{k}, \ldots x_{n}^{k}$ is a set of solutions that meet a certain precision. Otherwise, $x_{i}^{k+1}=x_{i}^{k}-\left.a^{k}\left(\partial \phi / \partial x_{i}\right)\right|_{x_{i}=x_{i}^{k}}$.

$$
\begin{aligned}
& a^{k}=\frac{\Phi\left(x_{1}^{k}, x_{2}^{k}, \Lambda, x_{n}^{k}\right)}{\left.\sum_{i=1}^{n}\left(\partial \Phi / \partial x_{j}\right)^{2}\right|_{x_{j}=x_{j}^{2}}}, \\
& \frac{\partial \Phi}{\partial x_{1}}=\frac{\Phi\left(x_{1}^{k}+h^{1}, x_{2}^{k}, \Lambda, x_{n}^{k}\right)-\Phi\left(x_{1}^{k}, x_{2}^{k}, \Lambda, x_{n}^{k}\right)}{h_{1}}, \\
& \frac{\partial \Phi}{\partial x_{2}}=\frac{\Phi\left(x_{1}^{k}, x_{2}^{k}+h_{2}, \Lambda, x_{n}^{k}\right)-\Phi\left(x_{1}^{k}, x_{2}^{k}, \Lambda, x_{n}^{k}\right)}{h_{2}},
\end{aligned}
$$

$\Lambda \Lambda$

$$
\begin{aligned}
& \frac{\partial \Phi}{\partial x_{n}}=\frac{\Phi\left(x_{1}^{k}, x_{2}^{k}, \Lambda, x_{n}^{k}+h_{n}\right)-\Phi\left(x_{1}^{k}, x_{2}^{k}, \Lambda, x_{n}^{k}\right)}{h_{n}}, \\
& h_{i}=H * x_{i}^{k}, \quad i=1,2, \Lambda, n .
\end{aligned}
$$

Step 4. Correct $x_{i}^{k+1}$ repeatedly until

$$
\left(\Phi_{1}^{k+1}, \Phi_{2}^{k+2}, \Lambda, \Phi_{n}^{k+1}\right)<\varepsilon
$$

The block diagram is shown in Figure 7.

After performing principal component analysis on the structural parameters of the flowmeter through characteristic model-based principal component analysis, the order of the influencing factors of differential pressure $\Delta \mathrm{P}$ of the flowmeter is obtained, and the parameter convergence curve is shown in Figure 8.

The specific results are as follows:

(1) The height of Static hole, $B$

(2) The distance from the centerline to the full pressure hole, $C$

(3) The angle of full pressure surface, $A$

(4) The distance from centerline to static pressure hole, $H$

3.3. Industrial Process Practical Application Verification. According to the characteristic model-based principal component analysis, the results of principal component analysis for the structural parameters of the flowmeter are tested in the flowmeter model built by the simulation software and verified in specific industrial applications.

We use the characteristic model-based principal component analysis method to find the four main influencing factors of the flowmeter pressure difference $\Delta P$ and optimize these four parameters to achieve the purpose of improving the measurement accuracy of the flowmeter and reducing the measurement repeatability. We verify the effectiveness of the algorithm.

(1) Increase the height of the static hole from 1.5 to 6

(2) Increase the distance from the centerline to the full pressure hole from 1.5 to 1.875

(3) Reduce the angle of full pressure surface from $70^{\circ}$ to $56^{\circ}$

(4) Increase the distance from the centerline to the static pressure hole from 1.5 to 1.875

The results of the actual industrial production process show that after the optimization of the flowmeter structure parameters, the measurement error of the flowmeter is reduced to $0.2 \%$, and the measurement repeatability is reduced to 0.06 . The specific actual data is shown in the table. In addition, it was verified in subsequent production that the optimized flowmeter structure parameters added 2 million yuan in economic benefits to the manufacturer. It proves the 


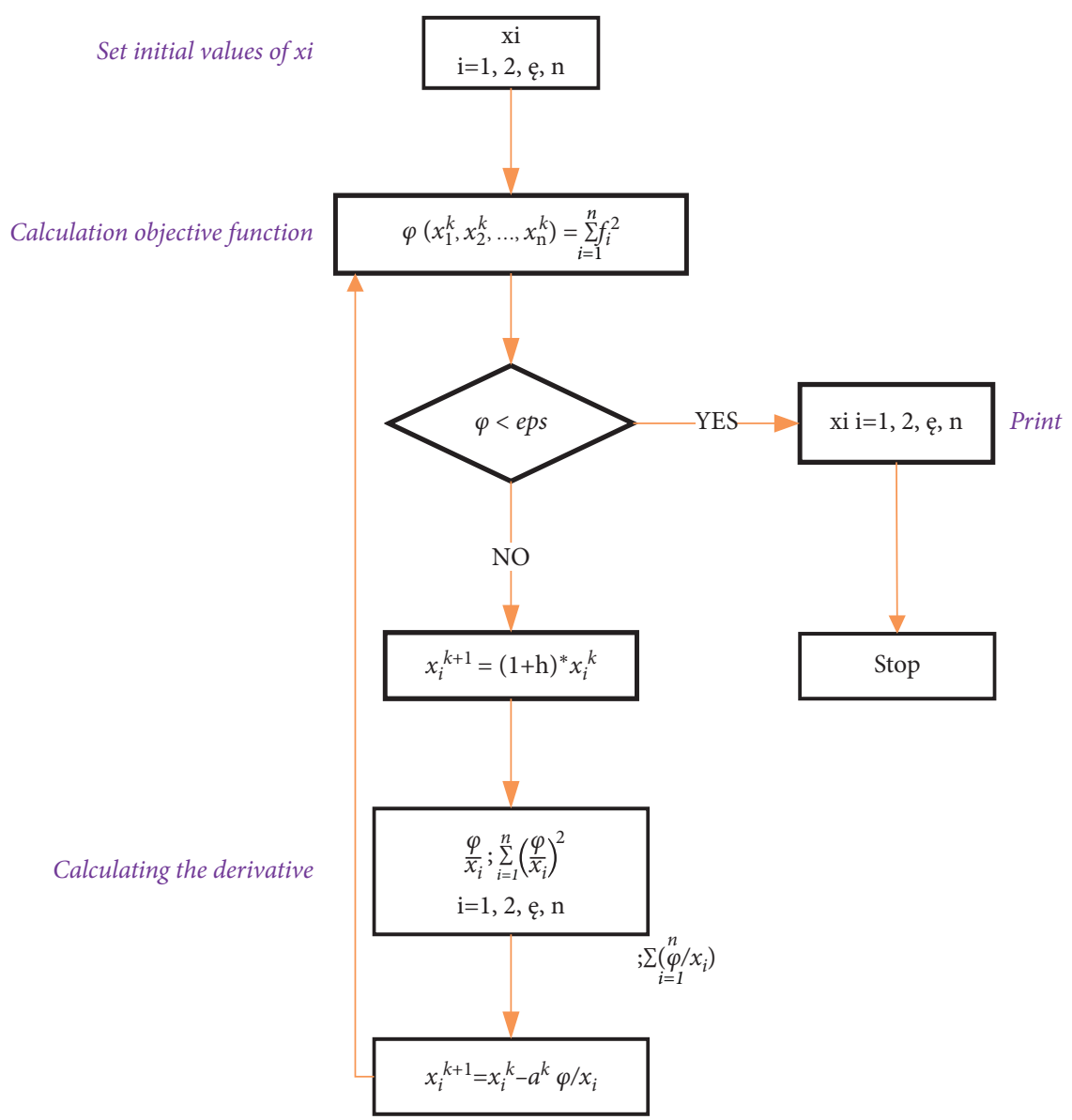

FIgURE 7: Block diagram of gradient method.

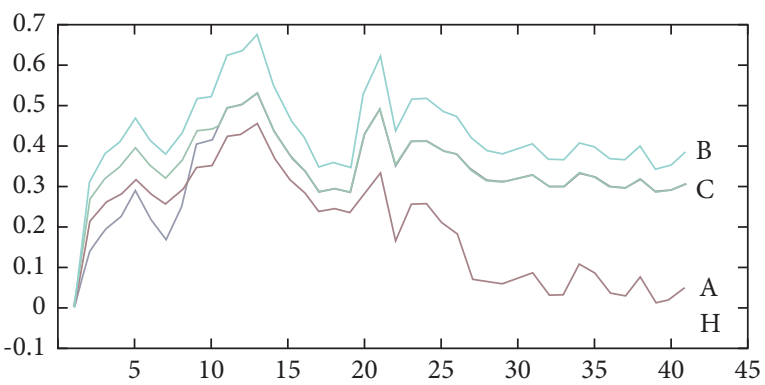

Figure 8: The parameter convergence curve.

effectiveness of characteristic model-based principal component analysis for the optimization of flowmeter parameters.

\section{Conclusion}

(1) This paper proposes a characteristic model-based principal component analysis method, introduces the characteristic model theory, and applies it to the optimization of flowmeter structure parameters.

(2) Take Bitoba flowmeter as an example, analyze its structural characteristics, use simulation software to simulate Bitoba flowmeter, and build a simulation model of Bitoba flowmeter.
(3) Use characteristic model-based principal component analysis to perform principal component analysis on the five structural parameters that affect the measurement accuracy of the flowmeter, and obtain the four structural parameters that have the greatest impact on them. After optimization of these four structural parameters, the Bitoba flowmeter's measurement error is reduced from $1 \%$ to $0.2 \%$, and the measurement repeatability is reduced from 0.3 to 0.06 , which increases economic benefits in industrial production.

It is proved that the characteristic model-based principal component analysis can well identify the nonlinear relationship existing in the system, and for the same structure, the system parameters will converge to a relatively fixed value, thus clearly distinguishing the principal component and its effect on the system and the size of the weight. For process control systems that cannot be accurately modeled widely in industrial processes, this method can decouple the coupling relationship between various variables through data identification and accurately find the principal elements of the system, providing an effective method for modeling such systems.

\section{Data Availability}

Data are unavailable. 


\section{Conflicts of Interest}

The authors declare that they have no conflicts of interest.

\section{References}

[1] M. S. Arulampalam, S. Maskell, N. Gordon, and T. Clapp, “A tutorial on particle filters for online nonlinear/non-Gaussian Bayesian tracking," IEEE Transactions on Signal Processing, vol. 50, no. 2, pp. 174-188, 2002.

[2] K. Fu, "Learning control systems and intelligent control systems: an intersection of artificial intelligent and automatic control," IEEE Transactions on Automatic Control, pp. 70-72, 1971.

[3] Y. Fu, Z. Gao, Y. Liu, A. Zhang, and X. Yin, “Actuator and sensor fault classification for wind turbine systems based on fast fourier transform and uncorrelated multi-linear principal component analysis techniques," Processes, vol. 8, no. 9, p. 1066, 2020.

[4] Z. Liu, G. Du, and L. Liu, "Study on internal flow field characteristics and measurement performance of momentum gas flowmeter," Acta Metrologica Sinica, vol. 40, no. 1, pp. 124-129, 2019.

[5] H. Yang, Y. Tu, and Y. Mao, "The ap-Hilbert method for phase difference estimation of Coriolis flowmeter," Chinese Journal of Scientific Instrument, vol. 40, no. 1, pp. 35-42, 2019.

[6] D. Park and J. S. Go, "Design of cyclone separator critical diameter model based on machine learning and CFD," Processes, vol. 8, p. 1521, 2020.

[7] W. Liu, K. Xu, J. Le et al., "Determination of two key parameters in the amplitude control of Coriolis flowmeter," Journal of Electronic Measurement and Instrument, vol. 32, no. 10, pp. 183-189, 2018.

[8] L. Sun, S. Dang, T. Zhang, and Y. Liu, "Experiment of flowmeter performance affected by flow stability," Journal of Tianjin University, vol. 51, no. 4, pp. 422-432, 2018.

[9] L. Wu, W. Yin, and C. Wang, "Development and experimental research of liquid fertilizer torque target flowmeter," Journal of Transduction Technology, vol. 30, no. 2, pp. 224229, 2017.

[10] H. M. Abd, O. R. Alomar and I. A. Mohamed, Effects of varying orifice diameter and Reynolds number on discharge coefficient and wall pressure," Flow Measurement and Instrumentation, 2019.

[11] Z. Wang, E. Andiroglu, G. Wang, and L. Song, "Accuracy improvement of virtual pump water flow meters using calibrated characteristics curves at various frequencies," Energy and Buildings, vol. 191, pp. 143-150, 2019.

[12] B. Oblak, S. Babnik, V. Erklavec-Zajec, B. Likozar, and A. Pohar, "Digital twinning process for stirred tank reactors/ separation unit operations through tandem experimental/ computational fluid dynamics (CFD) simulations," Processes, vol. 8, no. 11, p. 1511, 2020.

[13] J. Zhang, Di Wang, B. Xu, Su Qi, Z. Lu, and W. Wang, "Flow control of a proportional directional valve without the flow meter," Flow Measurement and Instrumentation, vol. 67, 2019.

[14] G. Wang, Z. Wang, and Li Song, "Uncertainty analysis for different virtual pump water flow meters," Science and Technology for the Built Environment, vol. 25, no. 3, 2019.

[15] D. V. Gradov, A. Saren, J. Kauppi, K. Ullakko, and T. Koiranen, "Auto-aspirated daf sparger study on flow hydrodynamics, bubble generation and aeration efficiency," Processes, vol. 8, p. 1498, 2020.
[16] S. Nasiruddin, S. N. Singh, S. V. Veeravalli, and S. Hegde, "Effect of vertex angle and vertex tip radius on the performance of V-cone flow meter using CFD," Measurement, vol. 138, pp. 536-544, 2019.

[17] G. Cortellessa and L. Iacomini, "A novel calibration system for heat flow meters: Experimental and numerical analysis," Measurement, vol. 144, pp. 105-117, 2019.

[18] S. Z. Islami rad and R. Gholipour Peyvandi, "A simple and inexpensive design for volume fraction prediction in threephase flow meter: single source-single detector," Flow Measurement and Instrumentation, vol. 69, Article ID 101587, 2019.

[19] M. Guilizzoni, G. Salvi, G. Sotgia, and L. P. M. Colombo, "Numerical simulation of oil-water two-phase flow in a horizontal duct with a Venturi flow meter[J]," Journal of Physics: Conference Series, vol. 1224, no. 1, 2019.

[20] G. Chiesa, C. Bertani, N. Falcone, A. Bersano, M. D. E. Salve, and B. Panella, "Horizontal air-water two-phase flow measurement using an electrical impedance probe and a venturi flow meter," Journal of Physics: Conference Series, vol. 1224, no. 1, 2019.

[21] Y. Liu, F. Yi, C. Zhou, and L. Tao, "Study on the influence of reflecting channel position on flow velocity characteristics of ultrasonic heat meter," IOP Conference Series: Materials Science and Engineering, vol. 544, no. 1, 2019.

[22] D. Duda, V. Yanovych, and V. Uruba, "An experimental study of turbulent mixing in channel flow past a grid," Processes, vol. 8, p. 1355, 2020.

[23] M. H. Roudi, J. Malekzadeh, M. Ebrahimi, A. Mirhaghi, and M. Shakeri, "Comparison between Emergency Severity Index plus peak flow meter and Emergency Severity Index in the dyspneic patients with chronic obstructive pulmonary disease: a randomized clinical trial," Turkish journal of emergency medicine, vol. 19, no. 2, pp. 68-72, 2019

[24] M. Fujita, K. Nagashima, S. Takahashi, K. Suzuki, T. Fujisawa, and A. Hata, "Handheld flow meter improves COPD detectability regardless of using a conventional questionnaire: A split-sample validation study," Respirology (Carlton, Vic.), vol. 25, no. 2, pp. 191-197, 2019.

[25] E. J. Jang, B. Nandram, Y. Ko, and D. H. Kim, "Small area estimation of receiver operating characteristic curves for ordinal data under stochastic ordering," Statistics in Medicine, vol. 39, no. 10, pp. 1514-1528, 2020. 